\title{
Factibilidad en la estimación ecocardiográfica de parámetros del ventrículo derecho en posición prono
}

\author{
Feasibility in the echocardiographic estimation of parameters of the right ventricle \\ in prone position
}

\author{
Luis E. Santos-Martínez ${ }^{1,2 *}$, Gastón Mendoza-Copaㄹ, Edgar García-Cruz¹, Rolando J. Álvarez-Álvarez', \\ Rafael E. Bucio-Reta1', Francisco J. González-Ruiz', Ángel Ramos-Enríquez', \\ Miguel Á. Hernández-Márquez ${ }^{1}$ y Francisco M. Baranda-Tovar ${ }^{1}$
}

${ }^{1}$ Departamento de Cuidados Intensivos Posquirúrgicos Cardiovasculares, Instituto Nacional de Cardiología Ignacio Chávez, Secretaría de Salubridad y Asistencia; ${ }^{2}$ Departamento de Hipertensión Pulmonar y Corazón Derecho, Unidad Médica de Alta Especialidad, Hospital de Cardiología del Centro Médico Nacional Siglo XXI, Instituto Mexicano del Seguro Social. Ciudad de México, México

\begin{abstract}
Resumen
Introducción: Los parámetros cardiacos ecocardiográficos en posición de decúbito prono usualmente se obtienen con sonda esofágica. Se desconoce la factibilidad de obtenerlos mediante aproximación transtorácica. Objetivo: Estimar la factibilidad para obtener parámetros del ventrículo derecho mediante ecocardiografía transtorácica en el sujeto en posición de decúbito prono. Métodos: Diseño piloto de serie de casos consecutivos sin enfermedad cardiopulmonar. Se acotaron variables demográficas, signos vitales y ecocardiográficas en posición decúbito ventral inicial, prono y ventral final. Los datos se muestran con promedios y desviaciones estándar, y frecuencias y porcentajes de acuerdo con la variable. La diferencia entre las posiciones se calculó con ANOVA de muestras repetidas y ajuste de Bonferroni. Se obtuvo la variabilidad intrasujetos mediante el procedimiento de Bland-Altman y su intervalo de confianza al 95\%. Resultados: Se estudiaron 50 sujetos, 44 (88\%) masculinos, edad $30 \pm 6$ años e índice de masa corporal $25.65 \pm 2.71 \mathrm{~kg} / \mathrm{m}^{2}$. El TAPSE (excursión sistólica del plano del anillo tricuspídeo) y la onda S' se midieron en el $100 \%$ de las veces. Los signos vitales y variables ecocardiográficas de acuerdo con la posición tuvieron diferencias en: frecuencia cardiaca ( $74 \pm 9$ vs. $77 \pm 9$ vs. $75 \pm 8$ lpm), saturación parcial de oxígeno ( $94.40 \pm 1.70$ vs. $96.64 \pm 1.79$ vs. $95.32 \pm 1.36 \%)$ y la presión arterial sistémica media (65.33 \pm 5.38 vs. $67.69 \pm 6.31$ vs. $65.29 \pm 5.62 \mathrm{mmHg})$; TAPSE (19.74 \pm 3.24 vs. $21.60 \pm 2.97$ vs. 19.44 vs. $2.84 \mathrm{~mm}$ ), diferencia media (sesgo) $0(2,-2.0)$ y onda $S^{\prime}(13.52 \pm 1.87$ vs. $15.02 \pm 2.09$ vs. $13.46 \pm 1.55 \mathrm{~cm} / \mathrm{s}$ ), diferencia media (sesgo) $-0.46(1.21,-2.14)$ respectivamente. Conclusión: En posición de decúbito prono es factible obtener parámetros del ventrículo derecho por ecocardiografía transtorácica.
\end{abstract}

Palabras clave: Posición del paciente. Ecocardiografía transtorácica. Ultrasonografía. Imagen diagnóstica. Hemodinamia.

\section{Abstract}

Background: Echocardiographic cardiac parameters in the prone position are usually obtained with an esophageal probe. The feasibility of obtaining them by means of a transthoracic approach is unknown. Objective: Estimating the feasibility to obtain parameters of the right ventricle by transthoracic echocardiography in prone position on the subject.

Correspondencia:

*Luis E. Santos-Martínez

E-mail: luis.santos@cardiologia.gob.mx
Disponible en internet: 07-02-2020 Arch Cardiol Mex. 2020;90(2):130-137 www.archivoscardiologia.com 1405-9940/@ 2019 Instituto Nacional de Cardiología Ignacio Chávez. Publicado por Permanyer. Este es un artículo open access bajo la licencia CC BY-NC-ND (http://creativecommons.org/licenses/by-nc-nd/4.0/). 
Methods: Pilot design of consecutive case series without cardiopulmonary disease. Demographic, vital signs and echocardiographic variables were defined in the ventral initial, prone and ventral final decubitus positions. The data are shown with averages and standard deviations, and frequencies and percentages according to the variable. The differences between the positions were calculated with ANOVA of repeated samples and adjustment of Bonferroni test. Intra-subject variability was obtained by the Bland-Altman procedure and its $95 \%$ confidence interval. Results: We studied 50 subjects, 44 (88\%) males, age $30 \pm 6$ years and body mass index $25.65 \pm 2.71 \mathrm{~kg} / \mathrm{m}^{2}$. Tricuspid annular plane systolic excursion (TAPSE) and S'-wave were measured $100 \%$ of the time. The vital signs and echocardiographic variables according to the position had differences in: heart rate ( $74 \pm 9 \mathrm{vs}$. $77 \pm 9 \mathrm{vs}$. $75 \pm 8$ beats $/ \mathrm{min})$, partial oxygen saturation $(94.40 \pm 1.70 \mathrm{vs}$. $96.64 \pm 1.79 \mathrm{vs} .95 .32 \pm 1.36 \%)$ and mean systemic blood pressure $(65.33 \pm 5.38 \mathrm{vs}$. $67.69 \pm 6.31$ vs. $65.29 \pm 5.62 \mathrm{mmHg}) ;$ TAPSE (19.74 \pm 3.24 vs. $21.60 \pm 2.97 \mathrm{vs} .19 .44 \pm 2.84 \mathrm{~mm})$, mean difference (bias) $0(2,-2.0)$ and $S^{\prime}$-wave (13.52 \pm 1.87 vs. $15.02 \pm 2.09$ vs. $\left.13.46 \pm 1.55 \mathrm{~cm} / \mathrm{s}\right)$, mean difference (bias) -0.46 $(1.21,-2.14)$ respectively. Conclusions: Obtaining right ventricle parameters by transthoracic ecocardiopraphy is feasible in the prone position.

Key words: Patient positioning. Transthoracic echocardiography. Ultrasonography. Diagnostic image. Hemodynamics.

\section{Introducción}

La posición de decúbito prono (DP) o de decúbito ventral es una posición corporal anatómica del cuerpo humano que se caracteriza por yacer acostado boca abajo manteniendo la cabeza de lado y el cuello en posición neutral.

Esta posición es de uso común en procedimientos medicoquirúrgicos, como en la nefrolitotomía ${ }^{1}$, colangiopancreatografía retrograda endoscópica ${ }^{2}$ y en cirugías de fosa posterior de la cabeza, cuello, vértebras, retroperitoneo y estructuras posteriores del cuerpo ante cirugía plástica ${ }^{3,4}$.

En las unidades de cuidados intensivos, la posición de DP se ha utilizado como parte del tratamiento del síndrome de dificultad respiratoria del adulto (SDRA) $)^{5-7}$. Con esta posición en estos pacientes se ha definido mejoría en la oxigenación, mecánica pulmonar, relación ventilación/perfusión $(\mathrm{V} / \mathrm{Q})$ pulmonar y como una maniobra de protección pulmonar ${ }^{5-7}$; el efecto en la mortalidad aún se cuestiona, aunque un metaanálisis que incluyó 12 trabajos y 2,129 pacientes, mostró disminución de la mortalidad con el uso de DP durante más de 12 horas $^{8}$.

En el SDRA el estudio y tratamiento se ha centrado en la evaluación del daño vascular pulmonar y las metas han sido la «apertura pulmonar» y el optimizar la saturación arterial de oxígeno ${ }^{9}$.

Con los nuevos conocimientos acerca de la fisiopatología en el SDRA y con mejores métodos no invasivos para evaluar el ventrículo derecho (VD) como lo hace la ecocardiografía transtorácica (ETT) los aspectos hemodinámicos han tomado mayor relevancia, específicamente el desarrollo de cor pulmonale agudo ${ }^{10}$, que parece estar involucrado en la mortalidad.
La evaluación por ETT usualmente se realiza en posición de decúbito dorsal (supino) o lateral, ya que no interfiere con la posición del transductor ${ }^{11,12}$; sin embargo, en posición de DP la pared anterior del tórax se encuentra obstruida y por ende tener ventanas útiles para hacer estimaciones del VD parecen estar limitado.

Por lo que en este trabajo como primera aproximación a la entidad estudiamos en posición de DP sujetos sin enfermedad cardiopulmonar, con la finalidad de conocer la factibilidad para tener ventanas acústicas adecuadas para determinar parámetros del VD con ETT.

\section{Métodos}

El trabajo se realizó en el Departamento de Cuidados Intensivos Posquirúrgicos Cardiovasculares (TPQ) del Instituto Nacional de Cardiología Ignacio Chávez, del 1 de marzo al 31 diciembre de 2018.

Diseño del estudio: piloto, serie de casos. Se estudiaron sujetos consecutivos que acudieron al departamento de TPQ y aceptaron participar.

Criterios de inclusión y exclusión: se incluyeron sujetos de ambos sexos, mayores de 18 años sin enfermedad cardiopulmonar. Se excluyeron sujetos con alteraciones en la ventana acústica. Se eliminaron aquellos con estudios incompletos o de mala calidad para análisis.

Para obtener la saturación parcial de oxígeno $\left(\mathrm{SpO}_{2}\right)$ se utilizó en todos el mismo oxímetro de pulso, marca Onyx ${ }^{\circledR}$ II Modelo 9550 (Nonin Medical INC., Plymouth, Minnesota, EE.UU.).

La presión arterial sistémica (PAS) se obtuvo con un esfigmomanométro neumático marca Phillips ${ }^{\circledR}$ MP 70 . 


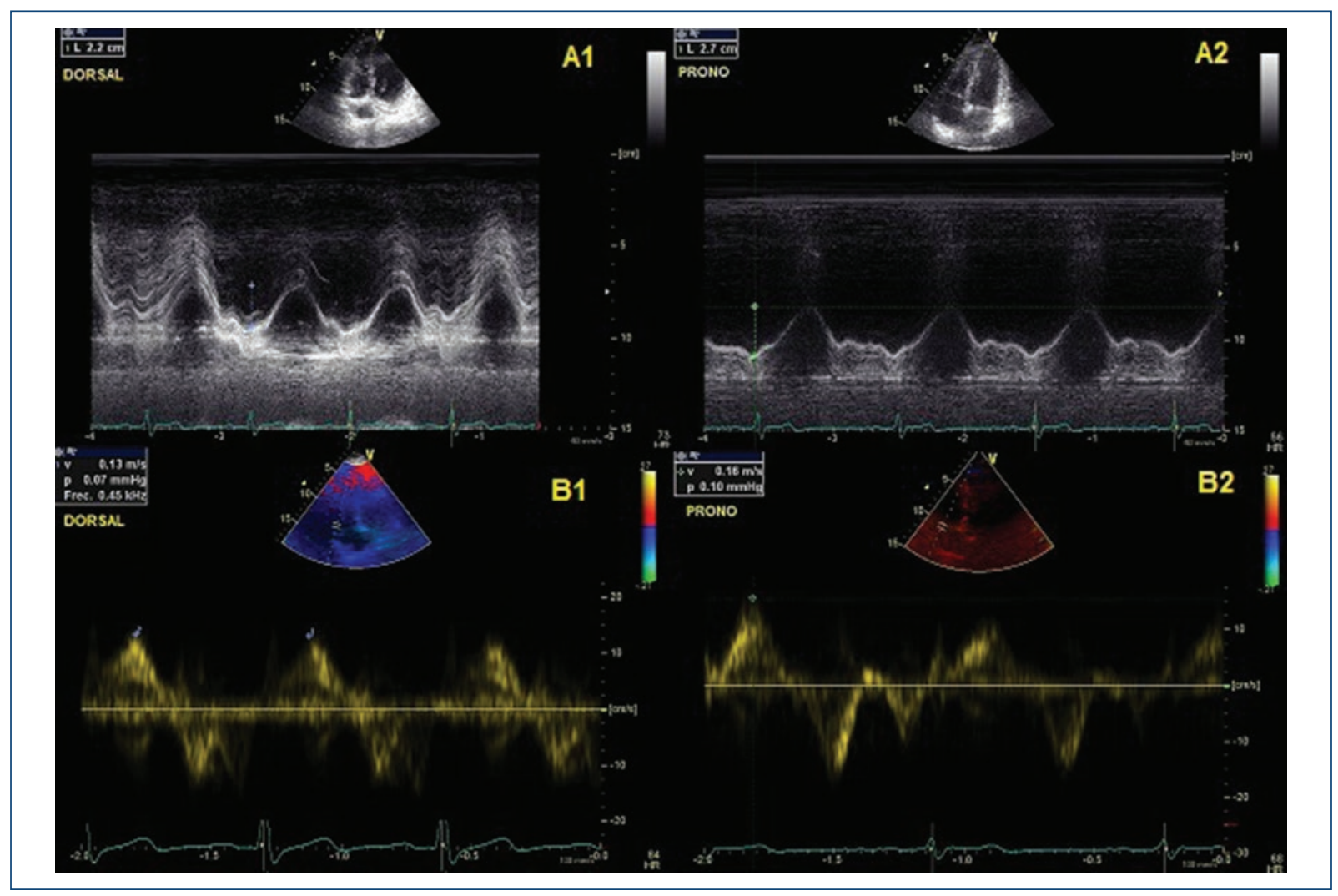

Figura 1. Imágenes ecocardiográficas de TAPSE (excursión sistólica del plano del anillo tricuspídeo) en decúbito dorsal (A1) decúbito prono (A2) y onda $S^{\prime}$ en decúbito dorsal (B1) y decúbito prono (B2). TAPSE decúbito dorsal y prono, en modo $\mathrm{M}$ y medida en mm. Onda $\mathrm{S}^{\prime}$, con Doppler tisular, medido en $\mathrm{cm} / \mathrm{seg}$.

Debido a la complexión delgada de todos los pacientes se utilizó en el brazo derecho el mismo brazalete estándar, con una cámara de $12 \times 26 \mathrm{~cm}$.

El equipo de ecocardiografía utilizado en todos los casos fue el sistema de ultrasonido cardiovascular VIVID ${ }^{\circledR}$ E9, con sonda cardiaca sectorial M5S-D ${ }^{\circledR}(G E$ Vingmed Ultrasound A/S, Norten, Noruega), transductor de 1.5-4.6 MHz, las imágenes se obtuvieron con armónicas tisulares. No se utilizó medio de contraste ecográfico para mejorar la visualización de los bordes endocárdicos.

Todos los parámetros ecocardiográficos fueron adquiridos por el mismo investigador (GMC); la segunda evaluación, con fines de establecer la concordancia intraobservador de los parámetros, fue realizada 24 horas después de estimar la primera.

Los parámetros ecocardiográficos se cuantificaron de acuerdo a las guías publicadas por la sociedad americana de ecocardiografía para la evaluación del corazón derecho 11,12: TAPSE (tricuspid annular plane systolic excursion, excursión sistólica del plano del aniIlo tricuspídeo) calculado en modo $\mathrm{M}$ en plano apical de cuatro cámaras, medido en milímetros (Fig. 1, A1 y A2); onda $\mathrm{S}^{1}$ tricuspídea, velocidad tisular sistólica del anillo tricuspídeo lateral, mediante Doppler tisular en plano apical cuatro cámaras medida en $\mathrm{cm} / \mathrm{s}$, (Fig. 1, B1 y B2); cambio del área fraccional (CAF, \%) del VD, obtenido del área telediastólica del VD en relación al área telesistólica del VD en proyección cuatro cámaras, medido en porcentaje; la presión sistólica de la arteria pulmonar (PAP-s) se calculó por medio del gradiente de insuficiencia tricuspídea (IT) mediante la ecuación de Bernoulli simplificada: IT ${ }^{2}$ x 4 + PAD (presión de aurícula derecha) interrogada en proyección cuatro cámaras con Doppler continuo, estimada en $\mathrm{mmHg}$; relación VD/ventrículo izquierdo (VI), cuantificada en proyección de cuatro cámaras en diástole.

La posición del sujeto en DP requirió que permaneciera acostado boca abajo manteniendo la cabeza de lado y el cuello en posición neutral, además se movilizó el brazo izquierdo en dirección superior (hacia la cabeza) y la colocación de una almohadilla de $10 \mathrm{~cm}$ de altura en la región infraclavicular ventral, lo que permitió tener una mejor imagen (Fig. 2). El transductor se 


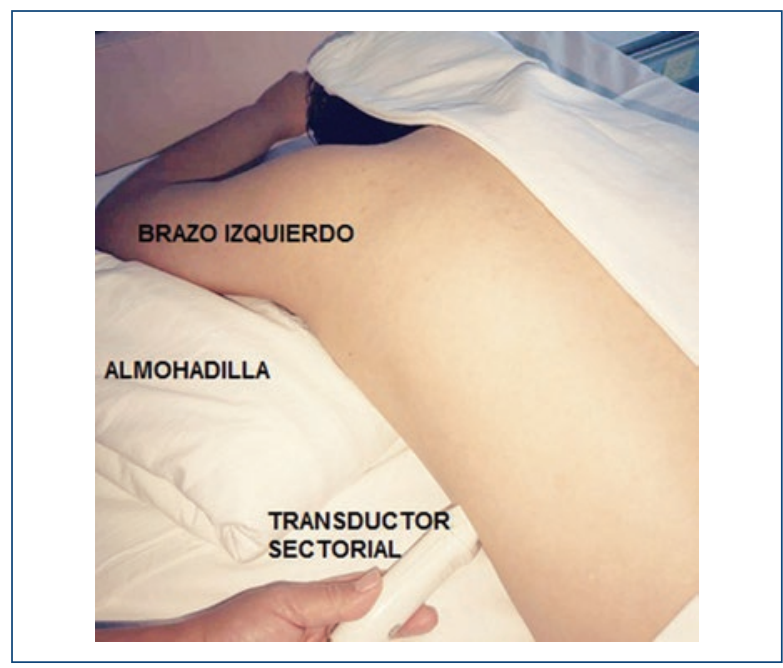

Figura 2. Posición de decúbito prono utilizada para obtener las variables ecocardiográficas.

colocó entre el $4 .^{\circ}$ y $5 .^{\circ}$ espacio intercostal izquierdo hacia la línea axilar anterior. Con esta posición se pudieron obtener en DP las variables mencionadas. Esta posición es una modificación de la posición de nadador utilizada en una Unidad de Cuidados Intensivos a propósito de la falta de ecocardiografía transesofágica ${ }^{13}$.

\section{Maniobra}

Una vez seleccionado al sujeto para estudio de acuerdo con los criterios de inclusión, se le colocó en decúbito dorsal (supino) y se procedió a determinar las variables ecocardiográficas mencionadas y los signos vitales; una vez realizadas, 10 minutos después se procedió a colocar al mismo sujeto en DP y se obtuvieron las mismas imágenes para su análisis ecocardiográfico y registrar los mismos signos vitales; finalmente el sujeto regresó a su posición de decúbito dorsal final y 10 minutos después se volvieron a estimar todas las variables. Un tercer participante, ajeno a las mediciones, recabó los resultados y los registró en la base de datos.

\section{Análisis estadístico}

Los datos se presentan como promedios \pm desviación estándar para los datos numéricos, y frecuencias y porcentajes para los nominales. El estudio de las diferencias entre grupos se realizó mediante ANOVA de muestras repetidas con ajuste de Bonferroni. Una $p<0.05$ fue considerada estadísticamente significativa. Se analizó la concordancia intrasujeto para los parámetros estimados del VD con el estadístico de Bland-Altman ${ }^{14}$ y su magnitud fue calculada mediante el coeficiente de correlación intraclase ${ }^{15}(\mathrm{CCl})$ y su intervalo de confianza al 95\% (IC 95\%).

Por ser un estudio piloto para valorar la factibilidad de estimar los parámetros, se utilizó una muestra de conveniencia con 50 sujetos.

El estudio fue aprobado por el Comité de investigación y ética del Instituto Nacional de Cardiología Ignacio Chávez, y todos los sujetos firmaron carta de consentimiento informado antes del estudio de ecocardiografía.

\section{Resultados}

Se estudiaron 50 sujetos sin enfermedad cardiopulmonar. La edad promedio fue $30 \pm 6$ años, la relación hombre/mujer fue $44 / 6$, el peso $77.33 \pm 10.85 \mathrm{~kg}$, talla $1.73 \pm 0.07 \mathrm{~m}$, superficie corporal $1.91 \pm 0.16 \mathrm{~m}^{2}$ y el índice de masa corporal $25.65 \pm 2.71 \mathrm{~kg} / \mathrm{m}^{2}$.

El comportamiento de los signos vitales de acuerdo con la posición interrogada se muestra en la tabla 1.

Las variables ecocardiográficas en decúbito dorsal y prono se acotan en la tabla 2. La frecuencia en que se pudieron adquirir las variables interrogadas de acuerdo con la posición de decúbito dorsal inicial, decúbito supino y decúbito dorsal final fueron respectivamente: TAPSE 50 (100\%), 50 (100\%), 50 (100\%); CAF: 50 (100\%), $44(88 \%), 50$ (100\%); ADVD $50(100 \%), 44(88 \%)$, 49 (98\%); ASVD: 50 (100\%), 44 (88\%), 49 (98\%); PAP-s 35 (70\%), 38 (76\%), 39 (78\%); onda S': 50 (100\%), $50(100 \%), 50(100 \%)$, y relación VD/VI: $50(100 \%)$, $46(92 \%), 50(100 \%)$.

La concordancia intraobservador para las variables ecocardiográficas en decúbito dorsal y supino se muestra en la tabla 3 . En la figura 3 se muestra la TAPSE como la variable ecocardiográfica con menor variabilidad en ambas posiciones.

\section{Discusión}

El objetivo del presente trabajo fue conocer mediante ecocardiografía transtorácica la factibilidad para estimar parámetros del VD relacionados con su función sistólica ${ }^{11,12}$ en la posición de DP en sujetos sin enfermedad cardiopulmonar.

La población estudiada tuvo una edad promedio de 30 años, fueron con mayor frecuencia de sexo masculino y el índice de masa corporal fue normal y sobrepeso; sin enfermedad cardiopulmonar.

El comportamiento de los signos vitales en la población estudiada fue como sigue: hubo pequeñas 
Tabla 1. Comportamiento de los signos vitales en posiciones de decúbito dorsal basal, prono y dorsal final

\begin{tabular}{|l|c|c|c|c|}
\hline & Decúbito dorsal $\mathbf{X} \pm \mathbf{D E}$ & Decúbito prono $\mathbf{X} \pm \mathbf{D E}$ & Decúbito dorsal $\mathbf{X} \pm \mathbf{D E}$ & $\mathbf{p}$ \\
\hline FC, Ipm & $74 \pm 9$ & $77 \pm 9$ & $75 \pm 8$ & $\begin{array}{c}1 \text { vs. } 2=0.014 \\
2 \text { vs. } 3=0.45 \\
1 \text { vs. } 3=0.789\end{array}$ \\
& & & & $\begin{array}{c}1 \text { vs. } 2=0.686 \\
2 \text { vs. } 3=1.00 \\
1 \text { vs. } 3=0.871\end{array}$ \\
\hline FR, rpm & $14 \pm 1$ & & $14 \pm 1$ & 1 vs. $2=0.001$ \\
& & $14 \pm 1$ & 2 vs. $3=0.001$ \\
1 vs. $3=0.001$
\end{tabular}

FC: frecuencia cardiaca; Ipm: latidos por minuto; FR: frecuencia respiratoria; rpm: respiraciones por minuto; PAS: presión arterial sistémica; S: sistólica; D: diastólica; $\mathrm{M}$ : media; $\mathrm{X}$ : media; DE: desviación estándar; $\mathrm{SpO}_{2}$ : saturación parcial de oxígeno.

Tabla 2. Comportamiento de las variables ecocardiográficas en posiciones de decúbito dorsal basal, prono y dorsal final

\begin{tabular}{|c|c|c|c|c|}
\hline & Decúbito dorsal $X_{ \pm} \mathrm{DE}$ & Decúbito prono $\mathrm{X} \pm \mathrm{DE}$ & Decúbito dorsal $X \pm D E$ & $\mathbf{p}$ \\
\hline TAPSE, mm & $19.74 \pm 3.24$ & $21.60 \pm 2.97$ & $19.44 \pm 2.84$ & $\begin{array}{l}1 \text { vs. } 2=0.001 \\
2 \text { vs. } 3=0.001 \\
1 \text { vs. } 3=0.749\end{array}$ \\
\hline Onda $\mathrm{S}^{\prime}, \mathrm{cm} / \mathrm{seg}$ & $13.52 \pm 1.87$ & $15.02 \pm 2.09$ & $13.46 \pm 1.55$ & $\begin{array}{c}1 \text { vs. } 2=0.001 \\
2 \text { vs. } 3=0.001 \\
1 \text { vs. } 3=1.00\end{array}$ \\
\hline CAF del VD, \% & $42.14 \pm 5.78$ & $45.73 \pm 5.72$ & $43.02 \pm 4.66$ & $\begin{array}{l}1 \text { vs. } 2=0.001 \\
2 \text { vs. } 3=0.004 \\
1 \text { vs. } 3=0.188\end{array}$ \\
\hline Área del VD en diástole, $\mathrm{cm}^{2}$ & $17.25 \pm 2.04$ & $17.58 \pm 2.18$ & $17.09 \pm 1.88$ & $\begin{array}{l}1 \text { vs. } 2=1.00 \\
2 \text { vs. } 3=0.312 \\
1 \text { vs. } 3=0.833\end{array}$ \\
\hline Área del VD en sístole, $\mathrm{cm}^{2}$ & $9.98 \pm 1.73$ & $9.58 \pm 1.63$ & $9.74 \pm 1.50$ & $\begin{array}{c}1 \text { vs. } 2=0.613 \\
2 \text { vs. } 3=1.00 \\
1 \text { vs. } 3=0.227\end{array}$ \\
\hline PSAP, mmHg & $16.40 \pm 6.01$ & $15.89 \pm 5.24$ & $15.74 \pm 4.69$ & $\begin{array}{l}1 \text { vs. } 2=1.00 \\
2 \text { vs. } 3=1.00 \\
1 \text { vs. } 3=0.775\end{array}$ \\
\hline VD/VI & $0.74 \pm 0.12$ & $0.76 \pm 0.11$ & $0.75 \pm 0.12$ & $\begin{array}{l}1 \text { vs. } 2=0.156 \\
2 \text { vs. } 3=0.154 \\
1 \text { vs. } 3=0.950\end{array}$ \\
\hline
\end{tabular}

X: media; DE: desviación estándar; TAPSE: excursión sistólica del plano del anillo tricuspídeo (tricuspid annular plane systolic excursion); CAF: cambio del área fraccional del ventrículo derecho; VD: ventrículo derecho; PSAP: presión sistólica de la arteria pulmonar; VD/VI: relación ventrículo derecho y ventrículo izquierdo.

diferencias en la frecuencia cardiaca $(\mathrm{FC})$ de la posición de decúbito ventral a DP y regresando a su FC basal al movilizarse el sujeto de nuevo a decúbito ventral, $\mathrm{p}<0.014$ (Tabla 1).
En cuanto a la frecuencia respiratoria (FR), no hubo modificaciones con los cambios de posición. No así en la $\mathrm{SpO}_{2}$, misma que incrementó al adoptar la posición de decúbito ventral a DP y regresando a su basal 
Tabla 3. Comportamiento de la diferencia media, sesgo, coeficiente de correlación intraclase e intervalo de confianza al $95 \%$ de las variables ecocardiográficas en decúbito dorsal y prono

\begin{tabular}{|l|c|c|c|c|}
\hline \multirow{2}{*}{} & \multicolumn{2}{|c|}{ Decúbito dorsal } & \multicolumn{2}{c|}{ Decúbito prono } \\
\cline { 2 - 5 } & Diferencia media (sesgo) & CCI (IC 95\%) & Diferencia media (sesgo) & CCI (IC 95\%) \\
\hline TAPSE, mm & $0(2,-2.0)$ & $0.976(0.950,0.988)$ & $0.1(2,-1.9)$ & $0.974(0.947,0.987)$ \\
\hline Onda S', cm/seg & $-0.46(1.21,-2.14)$ & $0.954(0.907,0.968)$ & $0.2(1.8,-1.4)$ & $0.963(0.923,0.982)$ \\
\hline FAC del VD, \% & $0.1(6,-5.8)$ & $0.944(0.882,0.973)$ & $0.9(8.3,-6.5)$ & $0.891(0.757,0.951)$ \\
\hline Área del VD en diástole, cm² & $0.23(1.44,-0.98)$ & $0.981(0.959,0.991)$ & $0.6(3.9,-2.7)$ & $0.862(0.691,0.938)$ \\
\hline Área del VD en sístole, cm² & $0.17(1.44,-1.11)$ & $0.973(0.944,0.987)$ & $0.4(2.6,-1.9)$ & $0.884(0.741,0.948)$ \\
\hline PSAP, mmHg & $-0.1(5.3,-5.6)$ & $0.954(0.892,0.981)$ & $-0.8(5.2,-6.9)$ & $0.945(0.878,0.976)$ \\
\hline VD/VI & $0.01(0.17,-0.14)$ & $0.856(0.698,0.932)$ & $0.02(0.19,-0.15)$ & $0.796(0.558,0.905)$ \\
\hline
\end{tabular}

CCI: coeficiente de correlación intraclase; IC 95\%: intervalo de confianza al 95\%; TAPSE: excursión sistólica del plano del anillo tricuspídeo (tricuspid annular plane systolic excursion); CAF: cambio del área fraccional del ventrículo derecho; VD: ventrículo derecho; PSAP: presión sistólica de la arteria pulmonar; VD/VI: relación ventrículo derecho y ventrículo izquierdo.

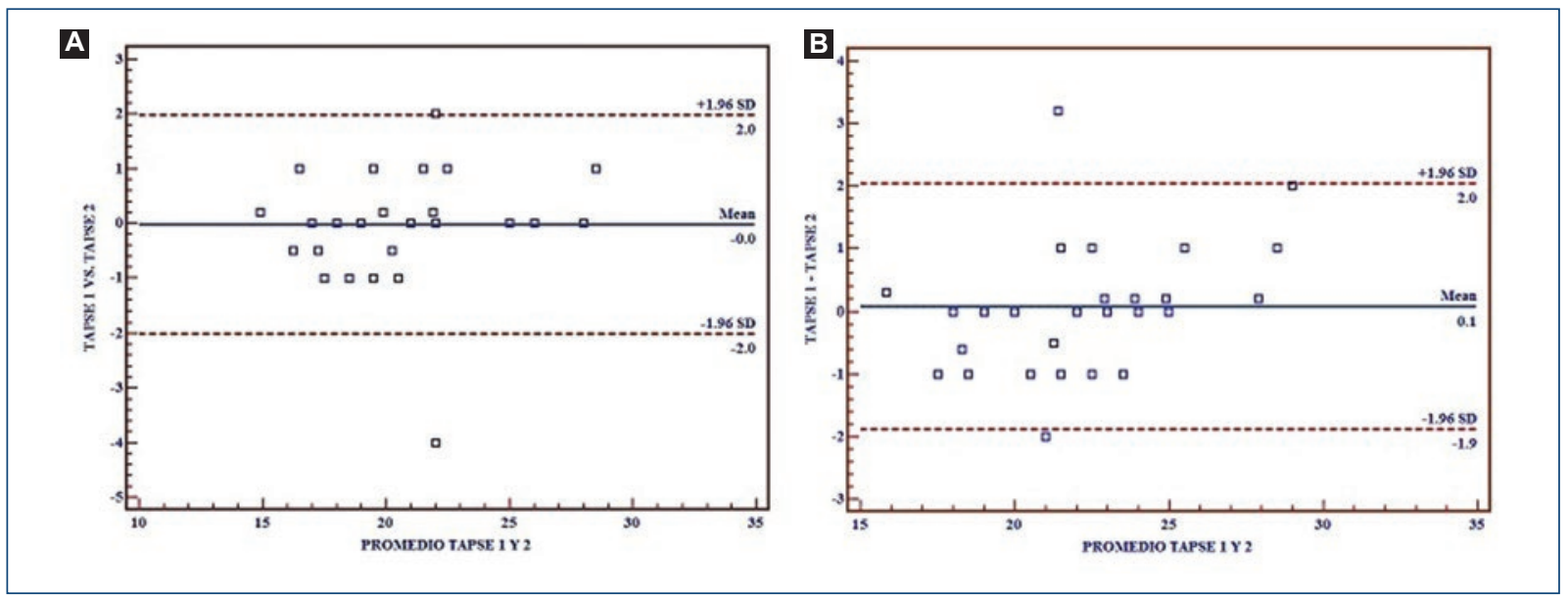

Figura 3. Diferencia media y sesgo del TAPSE (excursión sistólica del plano del anillo tricuspídeo) en decúbito dorsal (A) y prono (B).

SD: standard deviation; Mean: promedio.

al regresar al sujeto a decúbito ventral $(p=0.001)$ (Tabla 1).

En la PAS no se apreciaron cambios en las presiones sistólicas y diastólicas, sin embargo, la PAS media tuvo discretos incrementos de la posición de decúbito ventral inicial a DP $(p=0.037)$ y de DP a decúbito ventral $(p=0.050)$, con lo que se igualó a la PAS media inicial (Tabla 1).

Los cambios hemodinámicos estuvieron relacionados con los cambios de posición corporal, como se ha referido previamente en el ámbito de la anestesia al adoptar la posición de DP1-4,16,17. Estos traducen alteraciones en la precarga y la poscarga al corazón.
Asimismo, estos mismos cambios de posición corporal traen diferencias en los volúmenes pulmonares y por ende en la ventilación y la perfusión pulmonar, lo que se refleja en una mejor relación ventilación/perfusión pulmonar y su efecto en el intercambio gaseoso, que en nuestro trabajo fue evidenciado por una mejor $\mathrm{SpO}_{2}$ (mejor saturación de oxígeno).

Este trabajo no estuvo diseñado para describir la fisiopatología de los posibles cambios en los signos vitales o la hemodinámica en la posición prono, sin embargo, es posible hacer algunas consideraciones de lo que podría estar ocurriendo. Estas discretas alteraciones en los signos vitales parecen estar relacionadas 
con la compleja interacción que guardan la respiración y la circulación ${ }^{18-20}$.

Los cambios se han descrito con respiraciones a volumen corriente normal ${ }^{18}$ y no necesariamente al solo incremento de la FR, lo que apoyaría la falta de respuesta en la FR encontrada en nuestros resultados; en estados patológicos este comportamiento puede ser más evidente y deletéreo ${ }^{19}$.

Los cambios en las fases inspiratoria/espiratoria del volumen pulmonar y la presión intratorácica (PIT) durante el ciclo respiratorio normal alteran la precarga y la poscarga del corazón derecho e izquierdo, con lo que interactúan para variar el gasto cardiaco y la presión sanguínea con el ciclo respiratorio ${ }^{20}$.

Durante la fase inspiratoria de la respiración, mientras los alveolos se llenan de aire la PIT disminuye y favorece el retorno venoso sistémico hacia el tórax; el corazón derecho se llena y se bombea sangre hacia la arteria pulmonar, que se dilata para acomodar flujo. En dos a tres latidos la sangre llega al VI y la presión sanguínea aumenta al final de la inspiración y principio de la espiración, con lo que la PAS disminuye levemente en la inspiración y se incrementa durante la espiración; este efecto puede ser potenciado o contrarrestado por el aumento súbito de flujo sanguíneo al VI durante la inspiración ${ }^{18,21}$. Además el incremento transitorio en la PAS media con la posición de DP puede ser reflejo debido a la disminución del retorno venoso (precarga) inducida por la posición ${ }^{16,17}$.

En condiciones patológicas estas alteraciones pueden favorecer mecanismos de interdependencia ventricular ${ }^{18,19}$.

En posición de DP fue posible determinar los parámetros por ETT que se muestran en la tabla 2. Este estudio muestra además que es posible determinar algunos parámetros relacionados con la función sistólica del VD, lo que en teoría haría posible el determinarlos en sujetos con SDRA, lo cual será motivo de otro estudio.

La variabilidad intrasujetos de los índices interrogados es pequeña, específicamente para la TAPSE y la onda S', así como en el $100 \%$ de las veces se pudieron medir; esta pequeña variabilidad de la TAPSE, en otro contexto, ya ha sido informada ${ }^{22}$ previamente.

Estos parámetros ecocardiográficos del VD se modifican de acuerdo con los cambios de posición, específicamente al adoptar el DP, y pudieran estar relacionados con las alteraciones en la precarga que induce la modificación de la posición ${ }^{19-21}$.

\section{Limitaciones}

En este trabajo se cumplió el objetivo de conocer la factibilidad de obtener imágenes para el análisis de la función sistólica del VD. Sin embargo, la estimación se realizó en sujetos sin enfermedad cardiopulmonar y no en ventilación mecánica, como sucede en pacientes con SDRA ${ }^{5,6}$. Además la muestra estuvo restringida a pacientes jóvenes con índice de masa corporal máxima de sobrepeso.

No obstante lo mencionado, este trabajo muestra que al menos la posición de DP no es del todo una limitante para obtener dichas imágenes y estimar parámetros ecocardiográficos del VD. Lo cual hace de la ETT una herramienta no invasiva adecuada para valorar el momento en que un paciente con SDRA curse con cor pulmonale y optar por la pronación; y a la inversa también el momento en que desaparece el cor pulmonale y ya no es necesaria la posición de DP por mejoría del paciente.

\section{Conclusiones}

La ETT es una herramienta útil para determinar parámetros relacionados con la función sistólica del VD. En posición de DP es posible obtener imágenes por ETT para hacer un análisis congruente y factible de la función sistólica del VD.

Los cambios en los signos vitales y en los parámetros del VD estimado por ETT estuvieron relacionados con los cambios de posición.

\section{Financiamiento}

Los autores declaran que no recibieron ningún patrocinio para llevar a cabo este artículo.

\section{Conflicto de intereses}

Los autores declaran que no tienen ningún conflicto de interés.

\section{Responsabilidades éticas}

Protección de personas y animales. Los autores declaran que los procedimientos seguidos se conformaron a las normas éticas del comité de experimentación humana responsable y de acuerdo con la Asociación Médica Mundial y la Declaración de Helsinki. 
Confidencialidad de los datos. Los autores declaran que han seguido los protocolos de su centro de trabajo sobre la publicación de datos de pacientes.

Derecho a la privacidad y consentimiento informado. Los autores han obtenido el consentimiento informado de los pacientes y/o sujetos referidos en el artículo. Este documento obra en poder del autor de correspondencia.

\section{Bibliografía}

1. Mourmouris $P$, Berdempes M, Markopoulos $T$, Lazarou L, Tzelves L, Skolarikos A. Patient positioning during percutaneous nephrolithotomy: what is the current best practice? Res Rep Urol. 2018;10:189-93.

2. Mashiana HS, Jayaraj M, Mohan BP, Ohning G, Adler DG. Comparison of outcomes for supine vs. prone position ERCP: a systematic review and meta-analysis. Endosc Int Open. 2018;6(11):E1296-301.

3. DePasse JM, Palumbo MA, Haque M, Eberson CP, Daniels AH. Complications associated with prone positioning in elective spinal surgery. World J Orthop. 2015;6(3):351-9.

4. Kwee MM, Yik-Hong H, Rozen WM. The prone position during surgery and its complications: A systematic review and evidence-based guidelines. Int Surg. 2015;100:292-303.

5. Rialp-Cervera G. Efectos del decúbito prono en el síndrome de distrés respiratorio agudo (SDRA). Med Intensiva. 2003;27(7):481-7.

6. Martínez O, Nin N, Esteban A. Evidencias de la posición en decúbito prono para el tratamiento del síndrome de distrés respiratorio agudo: una puesta al día. Arch Bronconeumol. 2009;45(6):291-6.

7. Bos LD, Martin-Loeches I, Schultz MJ. ARDS: challenges in patient care and frontiers in research. Eur Respir Rev. 2018;27(147).
8. Munshi L, Del Sorbo L, Adhikari NKJ, Hodgson CL, Wunsch H, Meade MO et al. Prone position for acute respiratory distress syndrome. A systematic review and meta-analysis. Ann Am Thorac Soc. 2017;14(Suppl 4):S280-8.

9. Rawal G, Yadav S, Kumar R. acute respiratory distress syndrome: an update and review. J Transl Int Med. 2018;6(2):74-7.

10. Repessé $X$, Charron C, Vieillard-Baron A. Acute cor pulmonale in ARDS: rationale for protecting the right ventricle. Chest. 2015;147(1):259-65.

11. Rudski LG, Lai WW, Afilalo J, Hua L, Handschumacher MD, Chandrasekaran K. Guidelines for the echocardiographic assessment of the right heart in adults: a report from the American Society of Echocardiography. J Am Soc Echocardiogr. 2010;23(7):685-713.

12. Wu VC, Takeuchi M. Echocardiographic assessment of right ventricular systolic function. Cardiovasc Diagn Ther. 2018;8(1):70-9.

13. Ugalde D, Medel JN, Romero C, Cornejo R. Transthoracic cardiac ultrasound in prone position: a technique variation description. Intensive Care Med. 2018;44(6):986-7.

14. Bland JM, Altman DG. Statistical methods for assessing agreement between two methods of clinical measurement. Lancet. 1986;1:307-10.

15. Kramer MS, Feinstein AR. Clinical biostatistics LIV. The biostatistics of concordance. Clin Pharmacol Ther.1981;29:111-23.

16. Dharmavaram S, Jellish S, Russ P, Shea J, Mehmood R, Ghanayem A, et al. Effect of prone positioning systems on hemodynamic and cardiac function during lumbar spine surgery: An echocardiographic study. Spine. 2006;31:1388-93.

17. Edgcombe $\mathrm{H}$, Carter $\mathrm{K}$, Yarrow $\mathrm{S}$. Anaesthesia in the prone position. $\mathrm{Br}$ J Anaesth. 2008;100:165-83.

18. Cheifetz IM. Cardiorespiratory interactions: The relationship between mechanical ventilation and hemodynamics. Resp Care. 2014;59:1937-45.

19. Pinsky MR. Heart-lung interactions. Curr Opin Crit Care. 2007;13:528-31.

20. Bronicky RA, Anas NG. Cardiopulmonary interaction. Pediatr Crit Care. 2009;10:313-22.

21. Pinsky MR. Cardiovascular issues in respiratory care. Chest. 2005; 128:592S-97S.

22. Olmos-Temois SG, Santos-Martínez LE, Álvarez-Álvarez R, Gutiérrez-Delgado LG, Baranda-Tovar FM. Interobserver agreement on the echocardiographic parameters that estimate right ventricular systolic function in the early postoperative period of cardiac surgery. Med Intensiva. 2016;40:491-98. 